\title{
CAPACITY EXPANSION OF FIBER OPTIC INFRASTRUCTURE NETWORIS USING WAVELENGTH DIVISION MULTIPLEXING SYSTEMS
}

\author{
Amitava Dutta ${ }^{1}$, Peter Kubat ${ }^{2}$ \\ ${ }^{1}$ School of Management MSN 5F4, George Mason University \\ ${ }^{2}$ Verizon Laboratories, 40 Sylvan Rd.Waltham,MA 02451, USA \\ peter.kubat@verizon.com
}

\begin{abstract}
For the last decade, carriers have been aggressively installing optical fiber in their backbone and metro networks, replacing aging copper plant to improve capacity and network services. Nevertheless, explosive growth in Internet diffusion and advances in Internet based voice and data applications has resulted in unprecedented demand for additional bandwidth. To satisfy this demand in a cost effective manner, carriers are deploying Wavelength Division Multiplexing (WDM) systems to leverage existing optical infrastructure. A WDM system allows transmission of multiple wavelengths (lambdas) over a single fiber strand, substantially increasing bandwidth while incurring much less cost compared to the huge investment of adding new fiber cables to the fiber plant. This paper develops a cost minimization model for capacity expansion of optical networks using WDM systems. The model assumes that an existing optical network is in place and therefore takes into consideration the technological constraints on placing and operating new WDM components for capacity expansion. An LPbased heuristic solution procedure is proposed and tested on sample problems. Proposed improvements to the solution procedure are also discussed in conclusion.
\end{abstract}

Key words: Optical fiber networks, capacity planning, wavelength division multiplexing, linear programming, heuristics 


\section{INTRODUCTION}

Over the last decade, carriers have been aggressively installing optical fiber in their metro and long-haul backbone networks. However, the explosive growth in Internet diffusion and increasingly sophisticated voice and data applications has created unprecedented demands for additional bandwidth. Backbone data traffic has been increasing at a compound annual rate of more than $100 \%$ for several years [9]. To meet these demands, carriers are not only laying more fiber, but are also increasing the rates of data on each fiber strand, either by utilizing higher speeds (e.g., OC-192, OC-762) or multiplexing more wavelengths on one fiber strand. Currently, the most economical approach to relieving capacity exhaustion in existing fiber plant is to deploy wavelength-division multiplexing (WDM) systems $[2,7]$. However, since WDM systems and optical cross-connects are still expensive - they are cheaper only in relation to laying new fiber - the need for careful planning of WDM-based capacity expansion is paramount $[2,5,6]$. This paper develops a cost minimization model aimed at assisting such planning efforts.

\section{PROBLEM DESCRIPTION}

In optical networks, wavelengths (i.e., light of a single frequency) are the units of transport. Normally, a single wavelength (also known as a 'lambda') is carried directly over an optical fiber strand. WDM systems are built over the base fiber strands and essentially multiply fiber capacity by allowing many lambdas to share a single fiber strand (for details on WDM design and architecture see $[8,10])$. For example, an n:1 WDM system will carry $\mathrm{n}$ lambdas over a single fiber strand. Optical cross-connects, either opaque (OEO optical-electrical-optical) or transparent (OOO fully optical), in the nodes, switch (cross-connect) lambdas from one optical transport system to another [1]. Optical cross-connects, whether OEO or OOO, are equipped with frequency converters, so that lambdas can be considered to behave just like typical telecommunication channels. Namely, there is no requirement that the entire path traversed by a lambda have a single specific frequency (color).

Figure 1 illustrates the WDM-based optical capacity expansion problem. The seven nodes represent optical cross-connects; the lines connecting them represent optical fibers. The existing optical network may already have some WDM systems installed. The dotted lines in Figure 1 illustrate two such systems, between nodes $(5,6)$ and $(2,3)$, respectively. A new WDM system cannot be implemented using a fiber strand that is already part of an 
existing WDM system. Hence, on link $(2,6)$, the number of fiber strands available for implementing new WDM systems is two rather than three. However, existing WDM systems may have spare capacity, and this must be taken into account in planning capacity expansion.

In addition to their multiplexing capability, topological attributes of the way WDM systems are actually engineered also need to be considered explicitly in the planning process [2]. To illustrate, consider node pair $(1,7)$ in Figure 1. A WDM system installed between $(1,7)$ includes a pair of optical add/drop multiplexers placed at nodes 1 and 7 and connected to the respective cross-connects. However, an optical path, using available fiber strands, also has to be engineered between $(1,7)$, illustrated by the continuous heavy line between this node pair in Figure 1. Whatever the engineered paths may be, the routing topology of WDM systems needs to be considered in capacity expansion planning, as it impacts ability to route demand for lambdas between different OD pairs in the network. The major component of total WDM cost is the multiplexers installed at the end nodes, plus that of a few optical regenerators if needed.

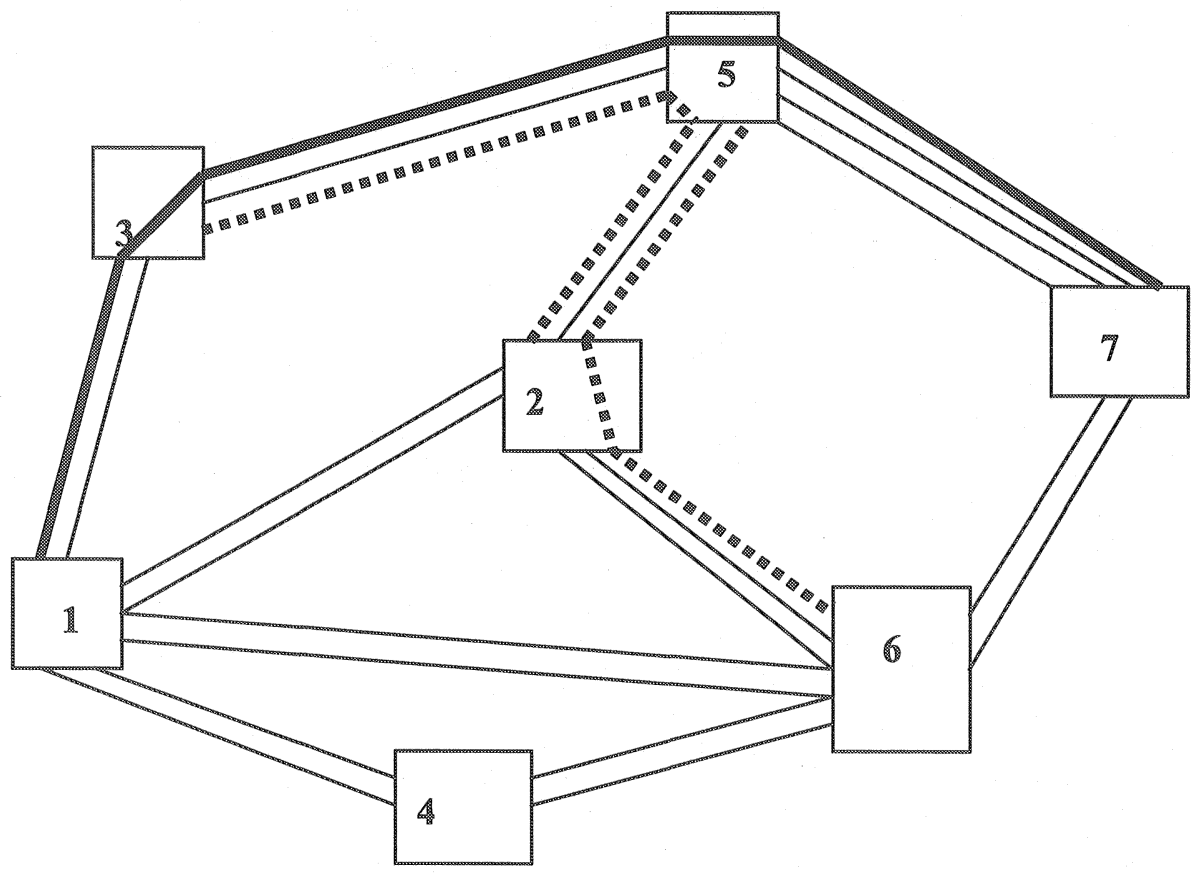

Figure 1. A WDM Based Optical Infrastructure Network

The objective of the capacity expansion problem addressed here is to increase the lambda carrying capacity of the optical network in a way such 
that all the demands for lambdas are routed and the total cost of the expansion is minimized. Specifically, the goal is:

(1) to decide where in the existing network WDM systems should be built and how many

(2) to find feasible routing for lambda-demand between all communicating node pairs

so that the total cost of network expansion is minimized.

\section{MODEL FORMULATION}

\section{Notation:}

$N$ - a set of nodes $i, j \in N$;

M - WDM multiplexing factor;

$\mathrm{K}-\subset \mathrm{NxN}$ of communicating origin-destination (OD) node pairs;

$D_{k}$ - demand requirement for $\mathrm{OD}$ pair $\mathrm{k} \in \mathrm{K}$, in lambdas;

$\mathrm{f}_{\mathrm{ijk}}$ - demand of communicating pair $\mathrm{k} \in \mathbb{K}$ routed onto link $\mathrm{ij}$;

$\mathrm{Q}_{\mathrm{ij}}$ - number of available fiber strands on link ij ;

$E_{i j}$ - existing capacity in lambdas either on the direct link $i j$ or on existing WDM systems between nodes $i$ and $j$;

$a_{i j} \quad$ - cost of building one WDM system between nodes $i$ and $j$;

$\mathrm{e}_{\mathrm{ji}}^{\mathrm{kl}}$ - indicator if a WDM system between nodes $\mathrm{k}$ and 1 is routed through link ij.

Namely,

$\mathrm{e}_{\mathrm{ji}}^{\mathrm{kl}}=\{1$, if WDM system for node pair kl uses a fiber on link $\mathrm{ij}$, 0 , otherwise

Decision variable:

$y_{i j}$ - number of WDM systems to be built between nodes $i$ and $j$. 


\section{Formulation:}

$\mathbb{P} 1: \quad \min \sum_{\mathrm{j}}\left\{\mathrm{a}_{\mathrm{ij}} \mathrm{y}_{\mathrm{ij}}\right\}$

subject to

$$
\sum_{j f_{i j}}{ }^{k} \sum_{j} f_{j i}^{k}=\left\{\begin{array}{l}
D_{k}, \text { if } O(k)=i \\
-D_{k}, \text { if } D(k)=i \quad \forall k \in \mathbb{K}, i \in N \\
0, \text { otherwise }
\end{array}\right.
$$

$\sum_{\mathrm{k}}\left(\mathrm{f}_{\mathrm{ij}}^{\mathrm{k}}+\mathrm{f}_{\mathrm{ji}}^{\mathrm{k}}\right) \leq M \mathrm{y}_{\mathrm{ij}}+\mathrm{E}_{\mathrm{ij}}, \quad \forall \mathrm{i}, \mathrm{j} \in \mathbb{N}$

$\sum_{\mathrm{k}, 1} \mathrm{e}_{\mathrm{ij}}^{\mathrm{kl}} \mathrm{y}_{\mathrm{kl}} \leq \mathrm{Q}_{\mathrm{ij}} \quad \forall \mathrm{i}, \mathrm{j} \in \mathrm{N}$,

$\mathrm{y}_{\mathrm{ij}}, \mathrm{f}_{\mathrm{ij}}^{\mathrm{k}} \geq 0$, integer $\forall \mathrm{i}, \mathrm{j} \in \mathrm{N}, \mathrm{k} \in \mathbb{K}$

Objective (1) minimizes the total cost of new WDM installations. Constraints (2) are standard flow balance equations; constraints (3) ensure that the total flow on a link is less then its capacity. Note that the right hand side of (3), which represents link capacity, includes existing spare capacity, $\mathrm{E}_{\mathrm{ij},}$ and the multiplexing factor, $\mathrm{M}$, of WDM systems. Constraints (4) relate the engineered path of DWM systems to the physical fiber layer as observed earlier in the paper. Since the indicators $\mathrm{e}_{\mathrm{ij}}^{\mathrm{kl}}$ de facto model "path engineering" of the WDM systems, constraints (4) ensure that available fiber capacity $\left(Q_{i j}\right)$ on a link is not exceeded. The indicator set, $e_{i j}{ }^{k l}$ for node pair $(k, 1)$ specifies where (i.e. on which particular link ij) fiber strands are needed for completing an optical path. The set is quite general and may be used to describe a variety of WDM topologies having different degrees of redundancy. It is a practical way to incorporate the engineering of the WDM systems and, in addition, makes the model manageable.

Also, note from constraints (3) that pre-existing capacity for lambda routing is distinguished from the fibers dedicated to building new WDMs by using different variables for each $-E_{i j}$ and $Q_{i j}$, respectively. This is appropriate, since fibers for new WDM must generally be of better quality, and as noted earlier, new WDMs cannot be built over existing WDMs.

\section{SOLUTION PROCEDURE}

Multi-commodity capacitated network problems, such as the one described above, are inherently hard to solve [4]. We are investigating a number of efficient heuristics for solving Problem P1 and our findings will 
be presented in a subsequent report. In this paper we concentrate on one promising heuristic only. It is based on a so-called "strong relaxation" (see [4] for details), which is obtained by dropping the integrality requirements in (5). The resulting linear programming (LP) problem is then solved. Integrality is achieved in an iterative manner by rounding fractional solutions one at a time and adding an additional constraint representing either a valid facet and/or restricting cut and resolving the LP. This process is repeated until a feasible and integer solution is obtained. This idea is summarized in the following pseudo-code, followed by a more detailed narrative of the different steps.

\section{Procedure WDM:}

Step 1. Relax integrality constraints on $\mathrm{f}_{\mathrm{ij}}^{\mathrm{k}}$ and $\mathrm{y}_{\mathrm{ij}}$ in P1. Call this problem $\mathbb{P} 1-\mathrm{R}$

Step 2. Solve P1-R using LP. Let $\left\{F^{*}, Y^{*}\right\}_{P 1-R}$ be the optimal solution $F^{*}$ and $Y^{*}$ are the flow and WDM vectors, respectively..

Step 3. Check $Y^{*}$ for integrality. $Y \Leftarrow Y^{*}$

Step 4. Define a set: $Y_{i n t}=\left(\left.y_{i j} \in Y\right|_{y_{i j}}\right.$ is integer $)$

Step 5. Define a set: $Y_{\text {frac }}=Y-Y_{\text {int }}$

Step 6. FOR $\left(y_{i j} \in Y_{\text {int }}\right) D O$;

Set lower-bound $\left(y_{i j}\right)$ \& upper-bound $\left(y_{i j}\right)=\left(y_{i j}\right)$ in P1-R

$\left[{ }^{*}\right.$ This constrains an integer value to remain integer ${ }^{*}$ ]

[* and fixing the value of the variable*]

\section{END FOR;}

Step 7. DO WHILE $\left(\mathrm{Y}_{\text {frac }} \neq \phi\right)$;

$7.1 \mathrm{y}^{\prime}{ }_{\mathrm{ij}}=\max \left(\mathrm{y}_{\mathrm{ij}} \in \mathrm{Y}_{\mathrm{frac}}\right)\left[{ }^{*}\right.$ choose the largest fractional value $\left.{ }^{*}\right]$

$7.2 \mathrm{y}^{+}{ }_{\mathrm{ij}}=$ integer $\left(\mathrm{y}^{\prime}{ }_{\mathrm{ij}}\right) \quad{ }^{*}$ make $\mathrm{y}^{\prime} \mathrm{ij}$ integer by rounding $\left.{ }^{*}\right]$

7.3 Set lower-bound $\left(\mathrm{y}^{+}{ }_{\mathrm{ij}}\right)$ \& upper-bound $\left(\mathrm{y}^{+}{ }_{\mathrm{ij}}\right)=\left(\mathrm{y}^{+}{ }_{\mathrm{ij}}\right)$ in $\mathrm{P} 1-\mathbb{R}$

7.4 Update: $Y_{\text {frac }}=Y_{\text {frac }}-\left(y^{+}{ }^{+}{ }^{j}\right)$

Update: $Y_{\text {int }}=Y_{\text {int }} \cup\left(y^{+}{ }_{i j}\right)$

7.5 Sumfrac $=\sum\left(y_{i j} \mid y_{i j} \in Y_{\text {frac }}\right)$

7.6 Add/modify constraint in P1-R:

$$
\sum\left(y_{i j} \mid y_{i j} \in Y_{\text {frac }}\right) \geq \text { ceiling (sumfrac) }
$$

7.7 LP: Solve P1-R using linear programming.

If solution infeasible, increase lower \& upper bound of $\mathrm{y}^{+}{ }_{\mathrm{ij}}$ by 1 and go to step $\mathbf{L P}$

7.8 Check $Y^{*}$ for integrality. $Y \Leftarrow Y^{*}$

$7.9 Y_{i n t}=\left(y_{i j} \in Y \mid y_{i j}\right.$ is integer $)$

END WHILE;

$$
Y_{\text {frac }}=Y-Y_{\text {int }}
$$

The first two steps of Procedure_WDM consist of relaxing integrality constraints (5) in problem $\mathrm{P} 1$ and solving the resulting LP. In the LP solution, it is possible that some of the $y_{i j}$ 's - which indicate how many 
WDMs are to be built between different OD pairs - may not be integer. Step 3 checks for integrality, while steps 4 and 5 separate integer and noninteger solutions into two sets $Y_{\text {int }}$ and $Y_{\text {frac }}$, respectively. Already found integer solutions are forced to remain fixed (step 6). The remainder of the heuristic, step 7, iteratively converts fractional $y_{i j}$ 's to integer values through rounding. In each iteration, the highest fractional $y_{\mathrm{ij}}$ is first chosen and rounded (steps $7.1 \&$ 7.2). The upper and lower bounds of this variable are then set to the same integer value to force integrality (step 7.3). In step 7.4, sets $Y_{\text {int }}$ and $Y_{\text {frac }}$ are revised to show that we have one more integer and one less fractional value. Once the highest fractional value has been rounded out, a restricting cut is added to the LP (steps $7.5 \& 7.6$ ). It says that the sum of the remaining fractional variables must be greater than the ceiling of this sum. For example, if $y_{13}, y_{15}$ and $y_{35}$ are the remaining fractional variables, with values $0.9,0.6$ and 1.2 respectively, this constraint would say $y_{13}+y_{15}$ $+y_{35} \geq$ ceiling $(0.9+0.6+1.2)$. Following rounding and addition of this restricting cut, the LP is resolved in step 7.7. The intuition behind this is that rounding introduces incremental capacity changes and it is prudent to redistribute flows to reflect these incremental changes before proceeding to round off the next fractional WDM variable. Step 7 repeats itself until all fractional variables from the original unrestricted LP have been converted to integer values - i.e. set $Y_{\text {frac }}=\phi$, as can be seen in the condition governing the WHILE loop of step 7.

\section{COMPUTATIONAL RESULTS}

Procedure WDM was coded in FORTRAN with routines from the IMSL library being called to solve the LP subproblems. Initial results are now presented using five and eight node problem sets, in Tables 1 and 2, respectively. The unit costs for installing WDM systems between the respective $O D$ pairs are shown in both tables; all $E_{\mathrm{ij}}=0$. Due to lack of space, the WDM indicator set, $\mathrm{e}_{\mathrm{ij}}^{\mathrm{kl}}$, could not be shown for either problem set. 
Table 1. Computation Results for 5-Node WDM Problem Set

\begin{tabular}{|c|c|c|c|c|c|c|c|c|c|c|c|c|c|}
\hline \multicolumn{14}{|c|}{5 NODE PROBLEM } \\
\hline $\begin{array}{l}\text { O-D } \\
\text { Pair }\end{array}$ & $\begin{array}{c}\text { Unit } \\
\text { WDM } \\
\text { cost }\end{array}$ & $\begin{array}{l}\mathrm{P} 1 \\
\mathrm{D}_{\mathrm{k}}\end{array}$ & $\begin{array}{l}\text { P1 } \\
\text { LP }\end{array}$ & $\begin{array}{l}\text { P1 } \\
\text { Heu }\end{array}$ & $\begin{array}{l}\mathrm{P} 2 \\
\mathrm{D}_{\mathrm{k}}\end{array}$ & $\begin{array}{l}\text { P3 } \\
D_{k}\end{array}$ & $\begin{array}{l}\text { P4 } \\
D_{k}\end{array}$ & $\begin{array}{l}\text { P5 } \\
D_{k}\end{array}$ & $\begin{array}{l}\text { P6 } \\
D_{k}\end{array}$ & $\begin{array}{l}\text { P7 } \\
D_{k}\end{array}$ & $\begin{array}{l}\text { P8 } \\
D_{k}\end{array}$ & $\begin{array}{l}\text { P9 } \\
D_{k}\end{array}$ & $\begin{array}{c}P 10 \\
D_{k}\end{array}$ \\
\hline$(1,2)$ & 5 & 6 & 0.6 & 1 & 7 & 5 & 5 & 7 & 3 & 3 & 8 & 6 & 8 \\
\hline$(1,3)$ & 5 & 6 & 0.6 & 1 & 2 & 10 & 2 & 7 & 11 & 9 & 5 & 6 & 0 \\
\hline$(1,4)$ & 7 & 5 & 0. & 1 & 1 & 4 & 3 & 6 & 7 & 11 & 9 & 1 & 8 \\
\hline$(1,5)$ & 8 & 5 & 0.5 & 0 & 6 & 6 & 12 & 11 & 5 & 4 & 6 & 3 & 7 \\
\hline$(2,3)$ & 9 & 0 & 0.0 & 0 & 2 & 4 & 11 & 4 & 1 & 7 & 12 & 9 & 0 \\
\hline$(2,4)$ & 100 & 3 & 0.0 & 0 & 10 & 6 & 5 & 6 & 1 & 11 & 7 & 12 & 10 \\
\hline$(2,5)$ & 8 & 1 & 0.4 & 0 & 8 & 6 & 13 & 11 & 2 & 8 & 0 & 11 & 11 \\
\hline$(3,4)$ & 4 & 1 & 0.2 & 1 & 3 & 11 & 8 & 3 & 11 & 0 & 9 & 0 & 9 \\
\hline$(3,5)$ & 50 & 1 & 0.0 & 0 & 10 & 7 & 7 & 9 & 6 & 1 & 6 & 8 & 9 \\
\hline$(4,5)$ & 2 & 1 & 0.5 & 1 & 11 & 4 & 9 & 7 & 10 & 9 & 5 & 4 & 7 \\
\hline \multirow[t]{2}{*}{$M=10$} & LP Cos & 18.5 & & & 39.5 & 38.9 & 50.6 & 47.3 & 29.4 & 44 & 43.6 & 45.3 & 46.1 \\
\hline & Heu Cos & 23 & & & 46 & 46 & 67 & 57 & 37 & 53 & 55 & 56 & 52 \\
\hline
\end{tabular}

The "LP Cost" reported in both tables is the objective function value obtained for the completely unrestricted linear programming solution from Step 2 of the procedure. The two gray shaded columns in Table 1 compare $\mathrm{y}_{\mathrm{ij}}$ values obtained from the LP and the heuristic procedure for one problem instance. The large number of fractional values in the LP relaxation suggests that it may not be a good lower bound against which to compare the heuristic solution. 
Table 2. Computation Results for 8-Node WDM Problem Set

\begin{tabular}{|c|c|c|c|c|c|c|}
\hline \multicolumn{7}{|c|}{$8 \mathrm{NODE}$ PROBLEM } \\
\hline \multirow[t]{2}{*}{$\begin{array}{l}\text { O-D } \\
\text { pair }\end{array}$} & \multirow[t]{2}{*}{$\begin{array}{l}\text { WDM } \\
\text { cost } \\
\text { (unis) }\end{array}$} & \multicolumn{5}{|c|}{ O-D demand (in lambdas) } \\
\hline & & $\# 1$ & $\# 2$ & $\# 3$ & 44 & \#5 \\
\hline $1-2$ & 5 & 5 & 8 & 7 & 3 & 2 \\
\hline $1-3$ & 5 & 7 & 8 & 0 & 12 & 6 \\
\hline 1.4 & 7 & 10 & 12 & 12 & 8 & 6 \\
\hline $1-5$ & 8 & 11 & 10 & 0 & 7 & 10 \\
\hline $1-6$ & 6 & 9 & 11 & 12 & 11 & 10 \\
\hline $1-7$ & 9 & 1 & 9 & 2 & 7 & 1 \\
\hline $1-8$ & 4 & 11 & 8 & 8 & 9 & 0 \\
\hline $2-3$ & 9 & 3 & 10 & 7 & 8 & 4 \\
\hline $2-4$ & 100 & 8 & 3 & 2 & 0 & 7 \\
\hline $2-5$ & 8 & 3 & 1 & 10 & 11 & 11 \\
\hline $2-6$ & 5 & 5 & 3 & 11 & 10 & 9 \\
\hline $2-7$ & 6 & 2 & 7 & 2 & 2 & 5 \\
\hline $2-8$ & 5 & 2 & 5 & 8 & 7 & 3 \\
\hline $3-4$ & 4 & 3 & 0 & 10 & 5 & 2 \\
\hline $3-5$ & 50 & 10 & 11 & 7 & 0 & 10 \\
\hline $3-6$ & 75 & 0 & 10 & 1 & 4 & 8 \\
\hline $3-7$ & 10 & 12 & 2 & 8 & 10 & 8 \\
\hline $3-8$ & 7 & 9 & 11 & 5 & 9 & 5 \\
\hline $4-5$ & 3 & 11 & 1 & 12 & 4 & 11 \\
\hline $4-6$ & 4 & 6 & 12 & 11 & 8 & 8 \\
\hline $4-7$ & 6 & 7 & 4 & 5 & 9 & 6 \\
\hline $4-8$ & 8 & 6 & 8 & 7 & 0 & 4 \\
\hline $5-6$ & 50 & 6 & 8 & 5 & 10 & 8 \\
\hline $5-7$ & 3 & 2 & 9 & 8 & 0 & 5 \\
\hline $5-8$ & 6 & 11 & 5 & 0 & 11 & 10 \\
\hline $6-7$ & 8 & 9 & 12 & 3 & 0 & 2 \\
\hline $6-8$ & 5 & 3 & 0 & 0 & 10 & 8 \\
\hline $7-8$ & 5 & 3 & 1 & 5 & 10 & 4 \\
\hline $\mathrm{LP} \operatorname{cost}$ & & 112.3 & 123.9 & 100.2 & 115.8 & 111.5 \\
\hline Heu Cost & & 143 & 152 & 119 & 139 & 139 \\
\hline$M=10$ & & & & & & \\
\hline
\end{tabular}

We are in process of developing tighter bounds based on Lagrange multipliers. However, our manual examination of integral solutions in the 5node case suggests that the heuristic is promising. For instance, the five WDM systems installed for Problem 1 in Table 1 are the five lowest cost ones that could have been installed. Some more 'back-of-the-envelope' calculations for Problem 1 in Table 1 may be illustrative of the heuristic's promise. Consider a cut that isolates node 1 from the rest of the 5-node 
network. The demand flowing across this cut is $(6+6+5+5)=22$ lambdas. The capacity of this cut, as generated by our heuristic, is $(1+1+1)=3$ WDMs or 30 lambdas since the WDM multiplexing factor is 10 . This is the lowest number of WDMs that could have been installed for this cut and still maintain feasibility. Due again to space restrictions, we do not show the flows of the commodities, but it appears that the heuristic distributes flows in a very efficient manner. WDM fill rates are high.

\section{CONCLUSIONS}

WDM systems are being widely deployed in optical infrastructure to expand capacity of fiber networks. Our formulation captures essential engineering aspects of WDM systems while remaining tractable. Initial tests of the LP-based heuristic solution reported here, are promising. Further tests of the heuristic are under way, using larger problem sets. Moreover, two other heuristics, one based on branch-and-bound and the other on Lagrangean relaxation, are under study..

\section{REFERENCES}

[1] Bentivoglio, F. and E. Iannone, "The Opaque Optical Network," Optical Network Magazine, Oct. 2000, pp 24-32.

[2] Cardwell, R. H., O.J. Wasem, and H. Kobrinski, "WDM Architectures and Economics in Metropolitan Areas," Optical Network Magazine, Vol. 1, no. 3, pp. 41-50, 2000.

[3] Cox, L. A. and J. Sanchez (Ryan), "Cost Savings from Optimized Packing and Grooming of Optical Circuits: Mesh versus Ring Comparisons," Optical Network Magazine, Vol. 2, no. 3, pp. 72-90, 2001.

[4] Gendron, B T. G. Crainic and A. Frangioni, "Multicommodity Capacitated Network Design," in Telecommunication Network Planning (B. Sanso, P. Soriano eds.) pp. 1-19, Kluver Academic Publishers, Boston 1999.

[5] Gryseels M., P. Demeester, W. Van Parys and P. Arijs, "Long-term planning of Optical Networks," 2001 OFC-Optical Fiber Communications Conference, pp. WZ2-1 to WZ2-3.

[6] Michael B. and R. Richardson, "The Lambda that Roared" Computer Telephony, Dec 2000 , pp. 38-62.

[7] Poudyal, V, R. H. Cardwell, O.J. Wasem, and J.E. Baran. "The Effects of Demand Level and Number of Wavelengths on the Economics of Metropolitan WDM," Proceedings of Nat. Fiber Optics Eng. Conference, pp. 1133-1144, 2001.

[8] Ramaswami R and K. N. Sivarajan "Optical Networks: A Practical Perspective." 1998, Morgan Kaufman Publishers, Inc. San Francisco.

[9] Riezenman M., Optical Nets Brace for Even Heavier Traffic, IEEE Computer, vol. 38, no. 1, Jan 2001, p44-46.

[10] Song, S. "DWDM and the Future Integrated Service Networks, "IEEE Canadian Review Spring 2000, pp. 5-7. 